\title{
MRI guided muscle biopsy confirmed polymyositis diagnosis in a patient with interstitial lung disease
}

J Lampa, I Nennesmo, H Einarsdottir, I Lundberg

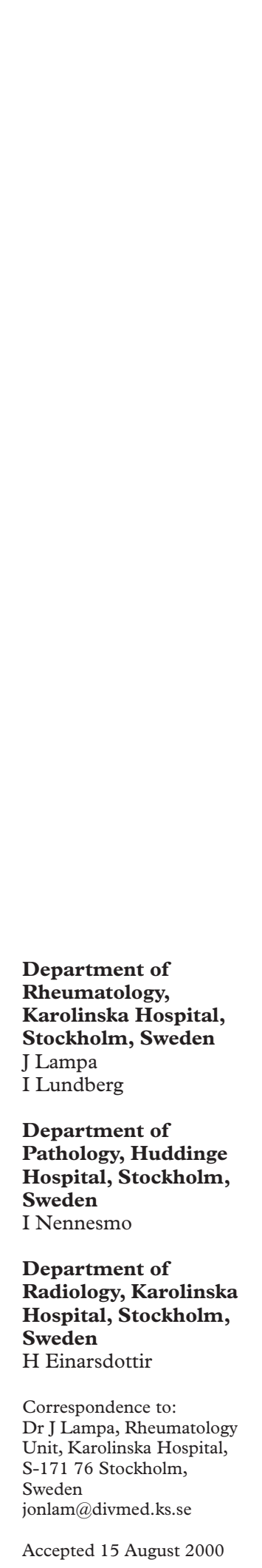

Abstract

Idiopathic inflammatory myopathies, such as polymyositis (PM), may present with general symptoms such as fever and fatigue and only minimal muscle weakness, making it difficult to make a definite diagnosis and provide adequate treatment. Here a case is described in which interstitial lung disease was the first and most prominent manifestation of $P M$. Later, when muscle weakness became apparent and inflammatory muscle disease was suspected the first muscle biopsy was non-diagnostic. However, magnetic resonance imaging (MRI) scans of the clinically weak thigh muscles showed high signal on $T_{2}$ weighted images, suggesting muscle inflammation more proximal to the first biopsy site. A second biopsy at this site disclosed typical histopathological findings for myositis. After treatment with prednisolone in combination with wh pulmonary and cyclophosphamide both pulmonary and uscle function improved.

Conclusion-MRI scans of muscles may be helpful in selection of a site for muscle biopsy in patients with suspected inflammatory myopathy when a first muscle biopsy turns out to be negative. Additionally, patients with interstitial lung disease of unknown cause should be tested for muscular function to exclude an associated inflammatory muscle disorder. (Ann Rheum Dis 2001;60:423-426)

Polymyositis (PM) is a subgroup of the idiopathic inflammatory myopathies (IIM), which are all characterised by symmetrical, proximal muscle weakness, decreased muscle endurance, and chronic inflammation in muscle tissue. ${ }^{1}$ Systemic features and other organ manifestations are not uncommon. ${ }^{2}$

Diagnosis is based upon symmetric proximal muscle weakness, an increase of serum levels of muscle enzymes, abnormal electromyogram, and the presence of an inflammatory infiltrate in muscle biopsy. ${ }^{3} \mathrm{~A}$ muscle biopsy is preferred to confirm diagnosis and to exclude other causes of muscle disorders. The typical histopathology of PM is the presence of degenerating and regenerating muscle fibres with inflammatory cells in and surrounding non-necrotic muscle cells. However, in some cases a muscle biopsy is negative. ${ }^{4}$ This might be due to a focal distribution of the inflammatory infiltrates, and some authors have advocated magnetic resonance imaging (MRI) as a routine investigation to select a biopsy site in order to increase the accuracy of a muscle biopsy. ${ }^{5}$ The diagnostic value of MRI in myositis is unknown, however, and only a limited number of cases have been reported in which MRI has been useful in selecting a muscle biopsy site. ${ }^{5}$ After MRI guided biopsy one case of treatment resistant IM turned out to have an inclusion body myositis (IBM) ${ }^{5}$ In another case MRI was se MRI was Pul

Here we describe a case in which a pulmonary manifestation was the first and most prominent symptom of the underlying inflammatory muscle disorder and in which muscle biopsy guided by MRI findings was helpful in establishing diagnosis.

\section{Case report}

A 60 year old man, previously healthy excep for an old injury in the plexus brachialis, presented in November 1997 at a primary care unit with muscle and joint pain, fever, and weight loss. Erythrocyte sedimentation rate (ESR) was $65 \mathrm{~mm} / 1 \mathrm{sth}$ and $\mathrm{C}$ reactive protein (CRP) $45 \mu \mathrm{cat} / \mathrm{(normal}$ value $<7$ ). Lactate ehydrogenase (LDH) was $15.8 \mu \mathrm{cat} / 1$ (norma value $<8 \mu \mathrm{cat} / \mathrm{l})$. A chest radiograph disclosed an interstitial infiltrate in the left lung. Pneumonia was suspected and the patient was treated with antibiotics for some weeks without effect.

During the following months he became dyspnoeic and a slight impaired pulmonary function was noted (total lung capacity (TLC) $74 \%$ of normal value and the transfer factor of the lung for carbon monoxide (TLCO) was reduced to $57 \%$ of the predicted value). Bronchoalveolar lavage showed a number of infirating inflammatory cells within the normal range. Polymerase chain reaction for Pneumocystis carinii from the lavage was positive and the patient was treated for three weeks with trimetoprim sulfamethoxazole in combination with $10 \mathrm{mg}$ prednisolone daily, with some reduction of the joint pain but without effect on the dyspnoea. At this time he had also developed severe muscle weakness and was de May 1998. On physical examination a symmetrical proximal and distal muscle weaknes was evident. The patient could barely hold his head upright and while standing up from sitting he had to support himself with his arms. The hands displayed hyperkeratosis, hyperpigmentation and he had then also developed arthritis with swelling of the metacarpophalangeal joints. 

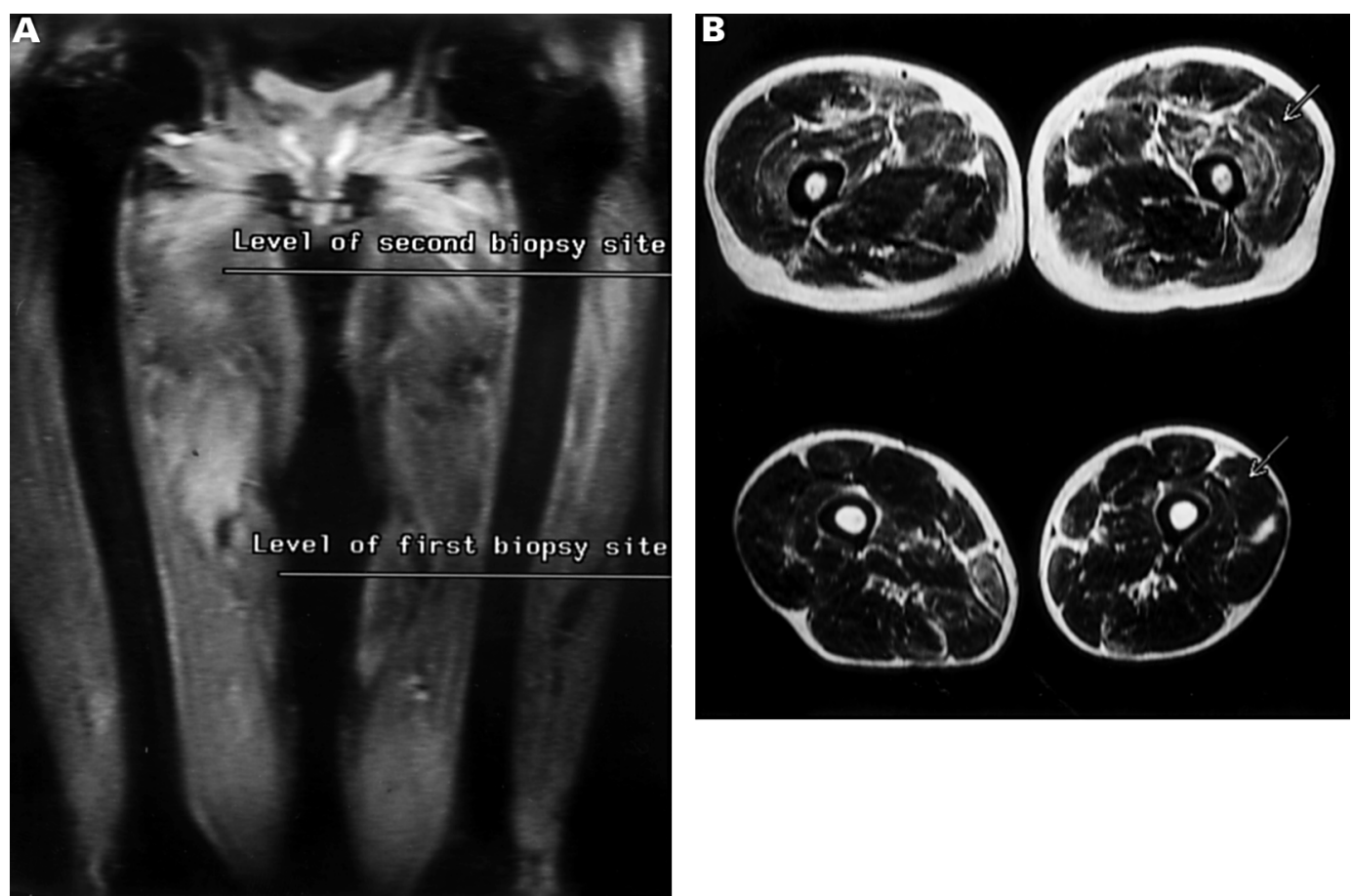

Figure 1 (A) Coronal MRI illustrating the approximate level of biopsy sites. (B) $T_{2}$ weighted axial images soon after admilammation in almost all muscles. The arrowe depicts the approximate location of the second biopsy. In the lowver slice only a slight signal increase is evident. The arrow denotes the approximate location of the first biopsy.

Laboratory investigations showed serum creatine kinase $>76.9 \mu \mathrm{cat} / \mathrm{l}$ (normal value $<3$ $\mu$ cat/l). Other serum muscle enzymes, aspartate

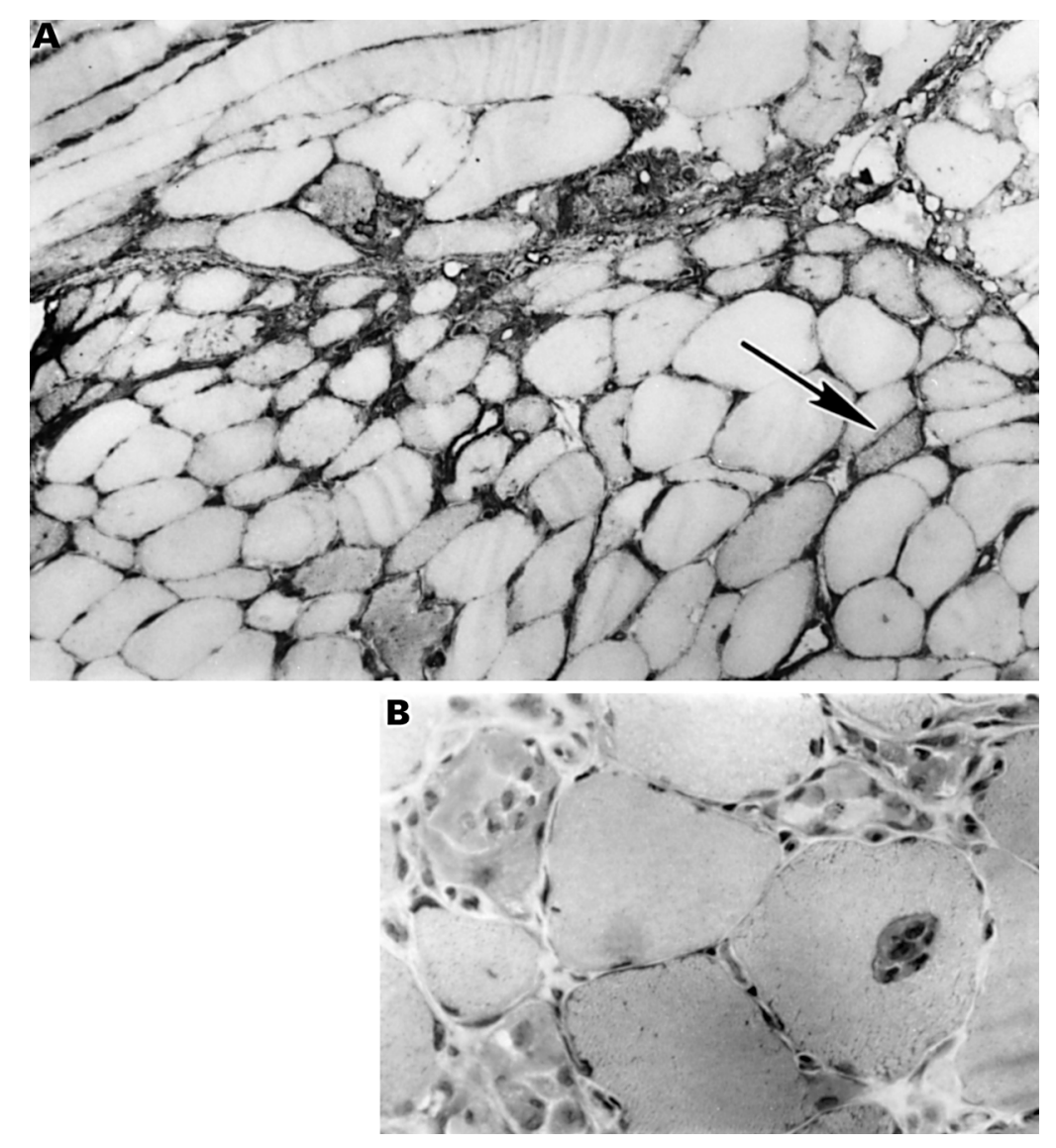

Figure 2 The second muscle biopsy from the left lateral vastus muscle.

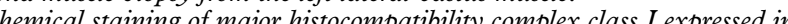
many muscle fibres (arrow). In this section prominent changes with numerous degenerating, Thescle fibres with a perifascicular diseribuion are seen. Note the infiltrates consisting of Tlymphocytes and macrophages in the perimysial mononuclear inflammatory cells. aminotransferase, alanine aminotransferase, $\mathrm{LDH}$, and myoglobin, were also increased. The ESR was $40 \mathrm{~mm} / 1 \mathrm{st} \mathrm{h}, \mathrm{CRP} 23 \mu \mathrm{cat} / 1$, haemoglobin $116 \mathrm{~g} / \mathrm{l}$, leucocyte count $20.4 \times 10^{9} / 1$ platelet count $503 \times 10^{9} / 1$, creatinine $90 \mu \mathrm{mol} / 1$ (normal value $<120 \mu \mathrm{mol} / \mathrm{l})$. High resolution computed tomography (HRCT) scan of the lungs displayed peripheral interstitial infiltrates.

A muscle biopsy from the vastus lateralis muscle disclosed minor pathological findings with occasional degenerating and regenerating fibres. An electromyogram displayed no changes related to muscle disease, but a discrete neuropathic pattern. However, the severe clinical symptoms still made IIM a likely diagnosis. MRI showed an increased signal on $\mathrm{T}_{2}$ weighted images most prominent in the proximal thigh muscles, indicating pronounced proximal inflammation (fig 1). Distally, where the firs biopsy specimen had been taken, only discrete signs of inflammation were evident (fig 1).

A second muscle biopsy more proximal in the vastus lateralis muscle showed tissue changes typical of inflammatory myopathy, including inflammatory infiltrates of mononuclear cells in the endomysium and with nonnecrotic fibres surrounded and invaded by inflammatory cells (fig 2). At the same time anti-Jo-1 antibodies were detected. A diagnosis of polymyositis with pulmonary involvement wo nitially treated with prednisolone $0.75 \mathrm{mg} / \mathrm{kg} /$ day and because of the severe pulmonary disease, cyclophosphamide $150 \mathrm{mg} /$ day.

Clinical improvement was already noticed after one week with subjectively decreased dyspnoea. One month later muscle function measured by a myositis index ${ }^{7}$ had improved. 


\begin{tabular}{l|l} 
Nov 1997 & Fever, weight loss \\
Jan 1998 & $\begin{array}{l}\text { Dyspnoea, impaired pulmonary } \\
\text { function } \\
\text { Feb }\end{array}$ \\
Mar & $\begin{array}{l}\text { Chest radiograph: infiltrates } \\
\text { Suspected Pneumocystis carinii } \\
\text { infection } \\
\text { Sulfamethoxazole and prednisolone }\end{array}$ \\
Apr & $\begin{array}{l}\text { Muscle weakness, patient referred to } \\
\text { Maymatology department } \\
\text { JRCT: interstitial lung changes }\end{array}$ \\
June & $\begin{array}{l}\text { Muscle biopsy 1 } \\
\text { MRI and muscle biopsy 2 } \\
\text { Prednisolone, cyclophosphamide } \\
\text { Decreased dyspnoea } \\
\text { Improved muscular function }\end{array}$ \\
Aug & $\begin{array}{l}\text { Regress of interstitial lung changes } \\
\text { on HRCT }\end{array}$ \\
Sep & $\begin{array}{l}\text { Oct } \\
\text { Nov }\end{array}$ \\
Figure 3 & Symptoms and investigations over time. HRCT
\end{tabular}

Figure 3 Symptoms and investigations
$=$ high resolution computed tomography.

Three months after starting treatment HRCT showed regression of the interstitial lung changes. After six months of treatment the muscle function was further improved, but pulmonary function tests were not changed (TLC $82 \%$ and TLCO $61 \%$ of predictive value). Figure 3 presents an overview of the clinical course.

\section{Discussion}

To our knowledge this is the first reported case in which MRI was useful in selection of a muscle biopsy site to confirm the diagnosis of polycle biopsy site to confirm the diagnosis of poly-
myositis in a patient with a severe interstitial myositis in a patient with a severe interstitial
lung disease. In this case fatigue, general symptoms, and dyspnoea initially predominated over the muscular symptoms. When muscle weakness progressed and an IIM was suspected the first muscle biopsy sample, although taken from the clinically weak thigh muscle, was non-diagnostic for IIM. However, a second biopsy taken proximally, the site being selected with guidance from the MRI, displayed typical histopathological findings for PM and the diagnosis was confirmed.

Inflammatory cell infiltrates and nonnecrotic muscle fibres invaded by mononuclear inflammatory cells are typical histopathological findings of PM and are regarded as a prerequisite for diagnosis of this condition. ${ }^{8} \mathrm{~A}$ diagnosis of possible or probable PM can still be made without typical changes in the muscle biopsy specimen according to the suggested criteria by Bohan and Peter. ${ }^{3}$ Normal muscle biopsies have been reported in 10-20\% of patients with IIM. Sampling error due to skip lesions has been suggested as one possible explanation for negative biopsies. ${ }^{4}$ Another reason for a negative biopsy, at least in some cases, may be treatment with corticosteroids before the biopsy sampling. In our case both explanations were considered as the cause of the first negative muscle biopsy. From the MRI scans, however, it was obvious that the earlier $10 \mathrm{mg}$ prednisolone regimen had not suppressed the muscle inflammation and that the negative biopsy was rather a result of the local distribution of the inflammation. Both the first and second biopsies were made within a period of two days, and therefore the contribution of a general disease progression as an explanation for the findings must be considered marginal.

MRI of muscle with $T_{1}$ and $T_{2}$ weighted images has been reported as a sensitive diagnostic tool in patients with myositis. Muscle changes in IIM as detected with MRI include oedema within and around muscles, muscle calcification, fatty infiltration, and fibrosis. ${ }^{9}$ The inflammatory lesions detected by MRI also correlate with disease activity, ${ }^{9}$ which MRI also correlate with disease activity, ${ }^{9}$ which
makes MRI a possible tool for follow up studes.

In the recently suggested revised classification criteria of IIM, positive MRI scans have been suggested for inclusion. ${ }^{10}$ The drawback of this technique as a routine diagnostic tool, however, is primarily the high costs that prevent the use of MRI scans as a screening tool for muscle inflammation. Furthermore, the inflammatory changes recorded are not specific for IIM and may therefore not replace a muscle biopsy in the diagnostic investigation. Besides, a muscle biopsy is still required to subclassify the IIM on histopathological grounds. $^{8}$

In this case general and pulmonary symptoms preceded the muscular weakness and careful diagnostic procedures for the pulmonary disease were undertaken, though without conclusive results. The patient was even treated with antibiotics for a suspected Pneumocystis carinii infection, which could later be excluded. Interstitial lung disease in PM or dermatomyositis occurs in about $5-30 \%$ of the cases, ${ }^{11}$ and in patients with anti-Jo-1 antibodies fibrosing alveolitis has been reported in as many as $50-100 \% .{ }^{12}$ The prognosis of these patients is generally poor with a five year survival of $40 \%{ }^{13}$ Fibrosing alveolitis has also been umber of patients with anti-Jo-1 antibodies and as the initial symptom in patients eventually developing myositis. ${ }^{14}$ An open lung biopsy was actually planned in our case when the muscular symptoms became prominent and an increased serum creatine kinase level made IM a likely diagnosis. To our surprise the muscle biopsy showed only slight non-specific findings. Although the observation of findings. Although the observation of hyperkeratosis and hyperpigmentation of the hands, clinical signs typical of "mechanic's hands", together with the positive anti-Jo-1 antibody would suffice for a diagnosis of Jo- 1 positive PM with interstitial lung disease, we still thought it to be of clinical importance to have 
muscle after the first biopsy turned out negative.

This case illustrates how muscle inflammation with moderate clinical muscle symptoms can be easily overlooked in patients with pronounced pulmonary disease. Thus a higher incidence of muscle inflammation in association with fibrosing alveolitis than hitherto reported may not be excluded, and clinical examination of muscle function should be included in these patients.

The presence of the anti-Jo-antibody seems to be a sensitive marker for lung disease,${ }^{14}$ and should in patients with primary interstitial lung disease raise the question of a possible
underlying inflammatory muscle disorder.

Our conclusion from this case is that in a patient with severe muscle symptoms and a negative muscle biopsy MRI should be performed in order to locate the inflammatory tissue changes.

We find it important to pursue the diagnostic procedures in order to get a histopathological confirmation of diagnosis. Firstly, because a delay in diagnosis due to a negative biopsy may result in a poor functional outcome. Secondly, a distinction between PM and IBM is not always evident on clinical grounds. Furthermore, we recommend that patients with interstitial lung disease of unknown cause should be tested for muscle function to exclude an associated inflammatory muscle disorder.

We thank associate professor Robert Harris for linguistic advice. Grant supports: The Swedish Rheumatism Association.
1 Dalakas MC. Polymyositis, dermatomyositis and inclusionbody myositis. N Engl J Med 1991;325:1487-98.

Interstitial lung disease in polymyositis and dermo Colby TV. tis. Clinical features and prognosis as correlated with histologic findings. Am Rev Respir Dis 1990; 141:727-33. 3 Bohan A, Peter JB. Polymyositis and dermatomyositis. N
Engl J Med 1975;292:344-7. 4 Bohan A, Peter JB, Bowman RI

assisted analysis of 153 patients Pearson CM. Computermatomyositis. Medicine (Baltimore) 1977;56:255-86

5 Sauty A, Rochat T, Schoch OD, Hamacher J, Kurt AM, Dannectic J 1997;10:2907-12.

6 Frazer AR, Miller RD. Interstitial pneumonitis in associaChest 1974

7 Engel AG, Arahata K. Mononuclear cells in myopathies: quantitation of functionally distinct subsets, recognition of eases, and implications for the pathogenesis of the differen inflammatory myopathies. Hum Pathol 1986;17:704-21. nary observations on the role of magnetic resonance imaging for polymyositis and dermatomyositis. Ann Rheum Dis

9 Fujino H, Kobayashi T, Goto I, Onitsuka H. Magnetic resonance imaging of the muscles in patients with polymyosi

and dermatomyositis. Muscle Nerve 1991;14:716-20.

Fujtake J, Ishikawa Y, Fujii H, Nishimura K, Hayakawa K,
Tatsuoka Y. Magnetic resonance imaging of skeletal muscles in the polymyositis. Muscle Nerve

1 Yoshida S, Akizuki M, Mimori T, Yamagata H, Inada S, Homma $M$. The precipitating antibody to an acidic nuclear protein antigen, the Jo-1, in connective tissue diseases. A nary fibrosis. Arthritis Rheum 1983;26:604-11.

12 Targoff IN, Miller FW, Medsger TA Jr, Oddis CV. Classifcation criteria for the idiopathic inflammatory myopathic

13 Josefson A, Romanus E, Carlsson J. A functional index in

4 Love LA, Leff RL, Fraser DD, Targoff IN, Dalakas M, Plo $\mathrm{PH}$, et al. A new approach to the classification of idiopathi inflammatory myopathy: myositis-specific autoantibodie timore) 1991;70:360-74. 